\title{
Transient Reduction in Reconfigurable Control Systems Utilizing Structure Dependence
}

\author{
Tamás Kovácsházy, Gábor Péceli, and Gyula Simon \\ Budapest University of Technology and Economics \\ Department of Measurement and Information Systems \\ H-1111, Múegyetem rkp. 9, Budapest, Hungary \\ \{khazy,peceli,simon\}@mit.bme.hu
}

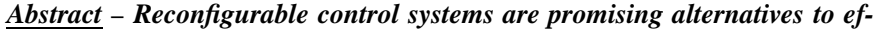
ficiently control complex time-variant and/or non-linear systems, such as vehicles, gas turbines, etc., which might require a certain level of faultoperational behavior, as well. Reconfigurable control systems are designed to be able to change the controller at run-time to react to failures, compensate the time-variant and/or nonlinear nature of plant, or accommodate changes in the control objectives. However, these changes in the plant and/or in the controller may cause intolerable transient. Management of these reconfiguration transients is an open issue. The dependence on the structure of the plant and the controller is identified as a key factor determining the reconfiguration transients. In this paper, some of the possibilities of reducing these transients are investigated. The results are demonstrated on a plant with two modes (operational and failure) controlled by properly designed PID controllers.
\end{abstract}

$\underline{\text { Keywords }}$ - Reconfigurable Control, Transient Management, Structure Dependence, Filter Structures

\section{INTRODUCTION}

Changing operational conditions of the plant asks for run-time adaptation or reconfiguration of the controller to keep the overall behavior within prescribed limits. Controller reconfigurations, however, may cause unacceptable transients in control loops. In this paper the issue of reducing transients due to controller reconfiguration and/or plant changes is investigated. The proposed solutions are based on the "management" of the state-variables of the controller by (1) selecting proper realization structures for the controller and/or (2) by modifying the state-variables of the controller. The properties of the proposed solutions are evaluated by simulation in a "test-bed" consisting of a plant with two modes (operational and failure) and a PID controller.

A conceptualization of a reconfigurable control system is a compound of a continuous-time plant, a discrete-time controller, a reconfiguration manager, and a supervisory subsystem. The block diagram of such a system is shown in Figure 1. The supervisory system detects the changes and faults of the plant, the actuators, the sensors, the controllers, including the underlying computing infrastructure, and works out a new configuration of the controller that assures proper operation under the given circumstances. In the majority of the cases, the supervisory sub-system can be considered as

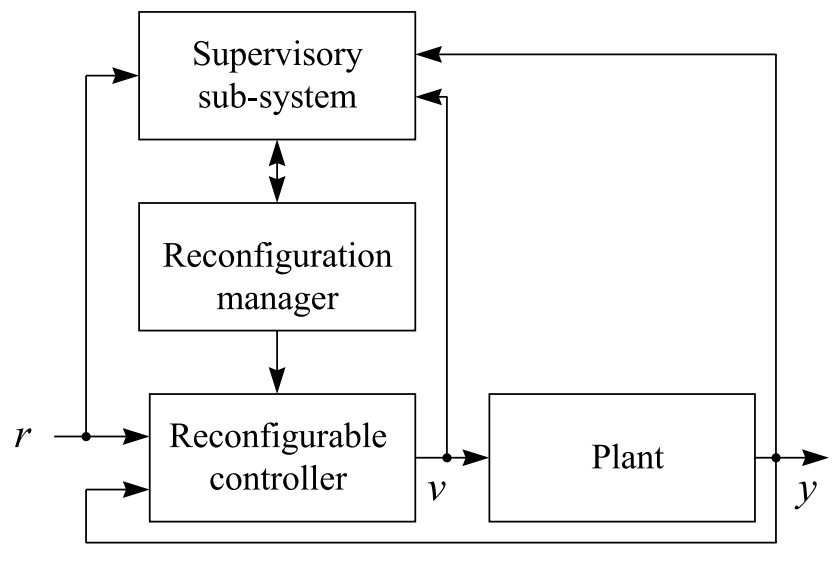

Fig. 1. Block diagram of a conceptual reconfigurable control system.

discrete-event system issuing reconfiguration commands to the controller through a reconfiguration manager. One of the primary tasks of the reconfiguration manager is to minimize the reconfiguration transients caused by the reconfiguration of the controller. As recent investigation show [1][2][3][4], without any interaction, reconfiguration transients might not be tolerated because of their possibly high amplitude and extreme dynamics. For this very reason, it is necessary to address the transient problem by introducing methods that guaranties acceptable transient behavior.

After a short introduction to the reconfigurable control in Section II, Section III gives some definitions required to investigate reconfiguration transients. We present structure dependence as a possible method to reduce transients in Section IV. A practical example is shown in Section $\mathrm{V}$, which demonstrates that the reconfiguration transients depend on the actual implementation of the controller, and that the transients can be reduced by selecting more suitable controller structure. Finally, conclusions are drawn in Section VI.

\section{RECONFIGURABLE CONTROL}

Reconfigurable control systems are in the center of research interest [1][5][6][7] because they offer a possibility to efficiently 
control time-variant and/or non-linear complex systems, such as vehicles, gas turbines, etc., which might require a certain level of fault-tolerance, as well. The reconfiguration transients are identified as a problem to be solved in all of the cited papers, but only [1] and [7] introduce reconfiguration methods that try to cope with the reconfiguration transients. The introduced methods are:

1. One step reconfiguration;

2. Multiple step reconfiguration with the gradual variation of the intermediate configurations using interpolation (series of one step reconfigurations);

3. Input cross-fading methods;

4. Output cross-fading methods;

5. State variable update methods;

6. Signal smoothing.

Recently, as a first step, structure dependence of the one-step reconfiguration was identified in open loop systems [8]. This result can be extended to transient reduction in control systems, as well.

\section{TRANSIENT MEASURES}

The optimal transition of a reconfigured system is highly environment and system dependent, therefore various transient definitions and transient measures exist. The appropriate one can be selected based on the requirements and transient tolerance of the environment, in which the system operates. The transient is defined generally as

$$
f_{t r}(n)=f(n)-f_{i d}(n),
$$

where $f_{t r}(n)$ is the transient of the variable, $f(n)$ is the observed variable in the investigated reconfigurable system, and $f_{i d}(n)$ is the same variable observed in an ideal reconfigurable system.

In addition to the definition of the transient, transient measures are required to compare and/or classify transients of contending alternatives for transient reduction. The energy of the transient, defined as

$$
\left\|\mathbf{f}_{t r}\right\|_{2}^{2}=\sum_{n=-\infty}^{\infty}\left|f_{t r}(n)\right|^{2},
$$

can be appropriate, or the absolute maximum of the transient, defined as

$$
\max _{n}\left|f_{t r}(n)\right|,
$$

is also a possible measure of choice in control systems, especially in systems with limited dynamics.

\section{STRUCTURE DEPENDENCE}

The one step reconfiguration method changes the coefficients of the system without modifying the internal states. The one step reconfiguration method utilizes the fact that the state variables store valuable information about the previous input samples, so we can, hopefully, continue operation of the new system producing smaller transients than transients produced by a systems reconfigured by other ways. Physical systems, in control systems the plant, preserve their state variables, i.e., stored energy can not be modified in infinitely small time without serious consequences. Therefore, plant reconfigurations, failures and intentional mode switches, can be considered as one step reconfigurations primarily.

Control systems are modeled as dynamic input-output mappings. For the linear case, they can be characterized by the impulse response in time-domain or by the transfer function in frequency-domain. Both descriptions are input-output descriptions, they do not specify how the internal processing is done. Both descriptions are only defined for $\mathbf{x}(0)=0$, where $\mathbf{x}$ is the state variable vector, so they can not be used as a system description in control schemes where the state variable vector differs from zero. The state variable description, however, for the linear case, in the form of

$$
\begin{gathered}
\mathbf{x}(n+1)=\mathbf{A x}(n)+\mathbf{B u}(n) \\
\mathbf{y}(n)+\mathbf{C x}(n)+\mathbf{D u}(n)
\end{gathered},
$$

is appropriate to investigate the transients of the one step reconfiguration method between consecutive reconfigurations. The transfer function $H(z)$ is invariant to the transformations

$$
(\mathbf{A}, \mathbf{B}, \mathbf{C}, \mathbf{D}) \leftarrow\left(\mathbf{T}^{-1} \mathbf{A} \mathbf{T T}^{-1} \mathbf{B}, \mathbf{C T}, \mathbf{D}\right),
$$

which means that infinite number of SVDs exist that realize the same transfer function. Various IIR filter structures [9][10] have been developed to utilize this invariance of the realized transfer function to the SVD to achieve better performance in IIR filters. It is shown in [8] that the internal structure also influences the transient behavior, and it is possible to minimize reconfiguration transients by selecting proper structures for implementation.

Applying the investigation of [8] directly to discrete control of continuous time plant is under consideration. It would require the transformation of one of the part, i.e., discrete or continuous, to the other domain of computation, with a transformation that is invariant not only to the input-output mappings, but to the internal energy relations. Unfortunately, the widely used discrete to continuous or continuous to discrete transformations do not satisfy the previously mentioned requirement, therefore the transient properties of the transformed system does not correspond directly to the original one.

Obviously, by evaluating the transient properties of the components of the control system, the plant and the controller, one can gain essential insight into the inner transient behavior of the entire control system, even if the complete characterization of the reconfiguration transient is not necessarily possible in the closed loop. 


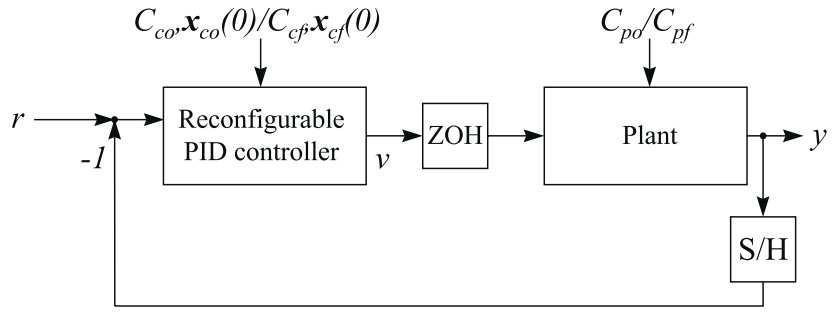

Fig. 2. Experimental setup.

Let us assume that the plant changes due to a fault, which causes unavoidable transients. Fault Detection and Isolation (FDI) algorithms use the difference of the nominal and observed system outputs, as defined in (1), to work out possible fault hypotheses [11]. To diagnose faults from transient, the FDI system must have a detailed built-in model of the plant that captures structural information, practically the information required to back-propagate the observed transient to possible changes in the internal energy flow in the physical system, to the actual fault. In essence, the FDI algorithms use the structure dependence of reconfiguration transient.

An immediate effect of the transient due to plant failure is the perturbation of the control loop. The controller tries to compensate the transient, but it is not designed for that task. In addition, the faulty plant demands the design of a new controller or the selection of an appropriate controller from stored controller alternatives to satisfy the control objectives. If the appropriate new controller is made available, it has been designed or selected, the actual controller have to be reconfigured to realize the new controller at run-time. This reconfiguration may result in additional transients, which can be reduced at will because we have full access to the controller realization, i.e., we can access and/or modify the signal flow graph, the internal states, the coefficients of the controller realization. Here we must note that we have very limited access to the plant, we can drive some of its inputs, we can measure some of its internal variables, and sometimes we can make quite limited changes in the plant.

\section{EXAMPLE}

The experimental simulated setup, shown in Figure 2, consists a continuous time plant and a discrete time PID controller. The failure of the plant is modeled by the transition from the operational configuration $C_{p o}$ to the failure configuration $C_{p f}$, and the repair is modeled by the transition from $C_{p f}$ to $C_{p o}$. The controller is to be reconfigured from its operational mode $C_{c o}$ to its failure mode $C_{c f}$ to properly control the failed plant, and from $C_{c f}$ to $C_{c o}$ when the plant is repaired. The state variables of the controller, $\mathbf{x}_{c o}$ and $\mathbf{x}_{c f}$, are accessible as shown on Figure 2, they can be read or written any time during operation. The properties of the used system configurations $\left(C_{p}\right.$ and $C_{c}$ pairs) are shown in Table I.
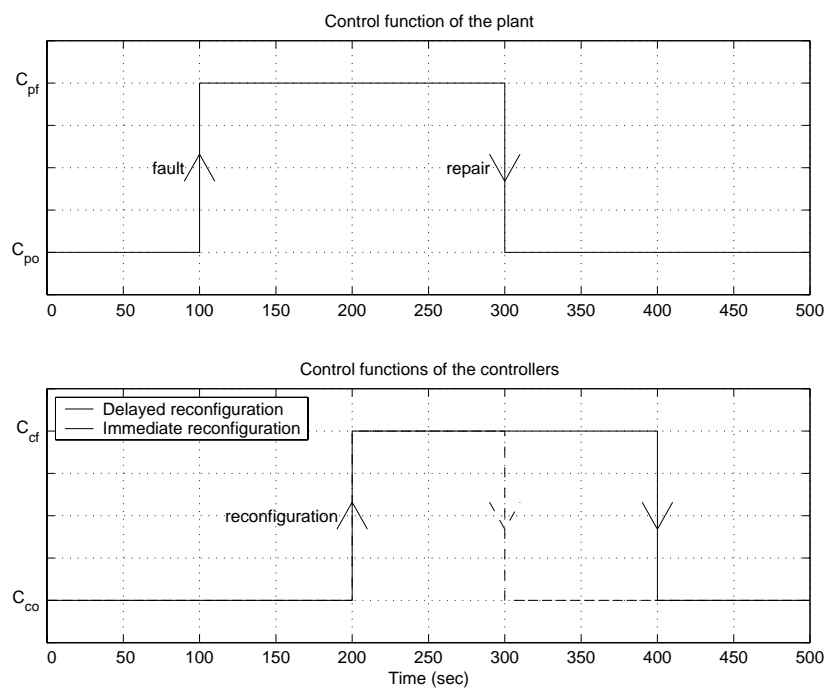

Fig. 3. Control functions for the plant and the controllers.

The failure of the plant is identified by the supervisory subsystem, typically from the transient [11] caused by the failure. In one scenario, this step is followed by the controller selection or design. In most of the cases, it is reasonable to assume that the reconfiguration of the controller occurs after the settling of the transient. The repair of the plant is done intentionally, which can be performed separately from the controller reconfiguration, or a joint repair of the plant and the reconfiguration of the controller is also possible. In the experimental setup, the changes of configuration are time driven. The control functions governing the configurations in the experimental setup are shown in Figure 3. Here we must note that the supervisory sub-system should take reconfiguration transients into account, and it should not initiate new reconfigurations ending in infinite repetitive sequence of reconfigurations.

For implementing discrete-time controller the so-called direct structure (see Figure 4(a) for signal flow graph) is widely because as it can be derived directly from its transfer function. This structure, however, has very bad reconfiguration transient properties as it was shown for IIR filters in [8]. The input scaled state-space structure, shown in Figure 4(b) and abbreviated as scaled SS in the figures, promises low transient in IIR filters [8]. The scaled state-space structure is used with minor modification as PID realization when the error signal and its derivative are both directly measured from the plant, but its application is possible in all other cases.

Another approach to reduce transients is the modification of the state variables of the controller. The simplest form of state variable modification is the zeroing of the state variables during the reconfiguration. More complex methods of computing new state variables for the new controller are possible, for example, by setting the state variables of the new controller to their future steady states. 


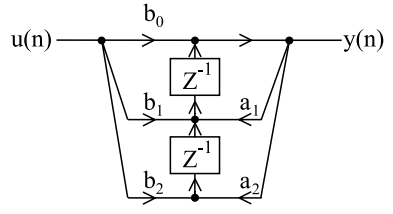

(a) (b)

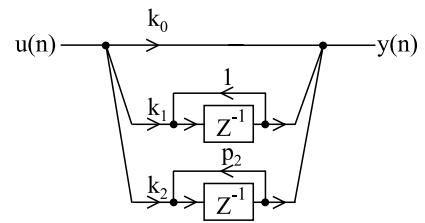

Fig. 4. Filter structures for controller realization. (a) Direct structure (b) State-space structure

In the experiments the reconfigurations are done using the onestep reconfiguration in a direct and a scaled state-space structure, and for reference a third experiment is included in which the state variables of the direct-form controller are zeroed.

First, the case of delayed reconfiguration is investigated. The controller and plant outputs are shown in Figure 5. As expected, the failure and repair of the plant cause transients invariant to the structure of the controller. On the other hand, the reconfiguration transients of the controller reconfiguration are structure and method dependent. The one-step reconfiguration of the direct structure and the state zeroing method are producing large transients compared to the transients of the one-step reconfiguration of the scaled state-space structure. If the reconfiguration of the scaled state-space controller is done when the whole system is in steady state practically no reconfiguration transients are produced. This preferable property is due to the state-space structure, because in steady state the input of the PID controller (the error signal) is 0 , and the change of the coefficients of all forward paths $k_{0}, k_{1}$, and $k_{2}$ do not effect the output of the controller, which does not hold for the direct structure.

The joint repair of plant and controller reconfiguration is investigated as the next experiment. The controller and plant outputs are shown in Figure 6. All experiments show large transients at 300 second. Unfortunately, state variables of both the direct and state-space controllers inherited from the failure configuration are not appropriate for the new controllers. To reduce the transients even in the case of joint plant repair and controller reconfiguration the state variables of the controller should be modified. By setting the state variables of the controllers in both the direct and state-space structures to its operational steady state we may achieve reduction of tran-

\begin{tabular}{|c|c|c|}
\cline { 2 - 3 } \multicolumn{1}{c|}{} & $C_{p o}$ & $C_{p f}$ \\
\hline$C_{c o}$ & no overshot & $\begin{array}{c}\text { over-compensated } \\
\text { large phase margin }\end{array}$ \\
\hline$C_{c f}$ & $\begin{array}{l}\text { under-compensated } \\
\text { small phase margin }\end{array}$ & no overshot \\
\hline
\end{tabular}

TABLE I

CLOSED LOOP PROPERTIES IN CASE OF PLANT AND CONTROLLER CONFIGURATIONS
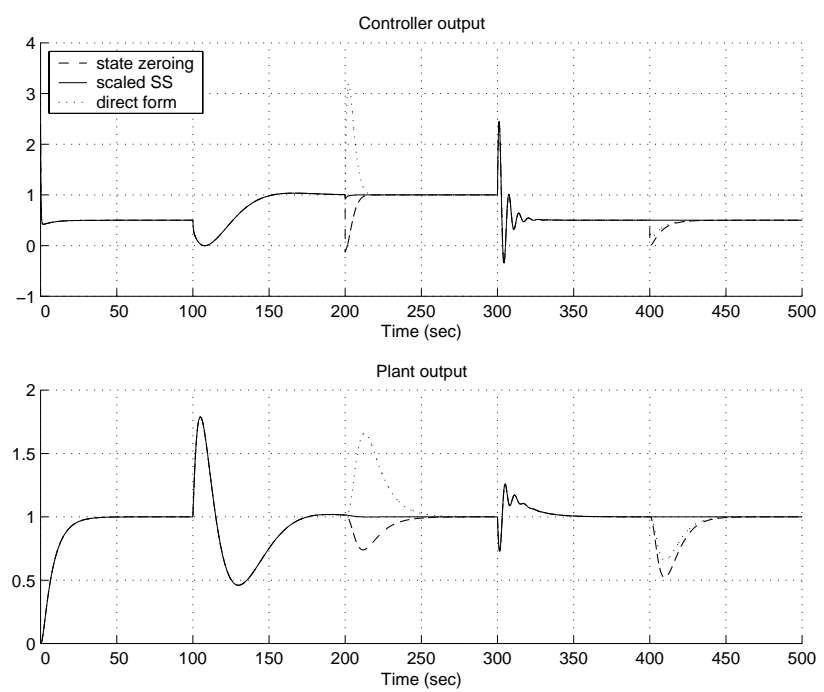

Fig. 5. Reconfiguration transients. The fault occurs at $100 \mathrm{sec}$, the controller is reconfigured according to the plant at $200 \mathrm{sec}$, at $300 \mathrm{sec}$ the plant is repaired, and finally at $400 \mathrm{sec}$ the controller is reconfigured according to the repaired plant.
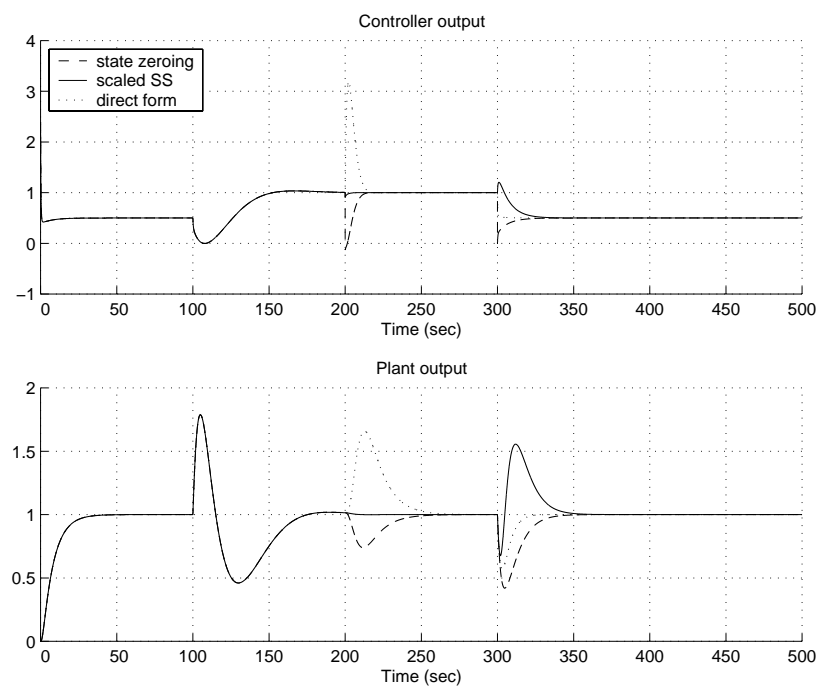

Fig. 6. Reconfiguration transients. The fault occurs at $100 \mathrm{sec}$, the controller is reconfigured according to the plant at $200 \mathrm{sec}$, at $300 \mathrm{sec}$ the plant is repaired parallel with the controller reconfiguration.

sients. The experiment is shown on Figure 7. The system utilizing the state-space controller shows moderate reduction of transients, while the transients of the direct structure controller are increased slightly. The appropriate method of computing the new state variables is under development, but limitation of state variable modification is identifiable, as we have no access to the state variables of the plant, which produces transients on its own.

\section{CONCLUSIONS}

Structure dependence is identified as a key factor in transient management of reconfigurable control systems. Using proper 

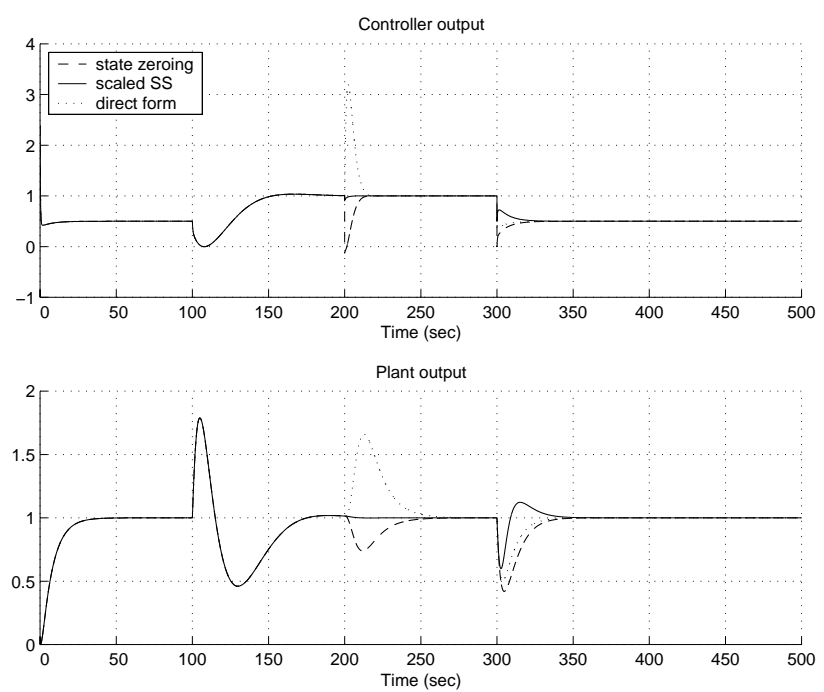

Fig. 7. Reconfiguration transients with state modification. The fault occurs at $100 \mathrm{sec}$, the controller is reconfigured according to the plant at $200 \mathrm{sec}$, at $300 \mathrm{sec}$ the plant is repaired parallel with the controller reconfiguration. The internal states of the controllers are set to their final steady states at $300 \mathrm{sec}$.

structure for controller realization guarantees no transient if the reconfiguration is performed in steady state of the system, and assures smaller transients than other structures for small disturbances. Transient property of the joint reconfiguration of the plant and the controller is an open question, but proper initialization of the state-variables of the controller can reduce transients.

\section{ACKNOWLEDGMENTS}

The research described here was sponsored, in part, by the Defense Advanced Research Projects Agency (DARPA) (US) under agreement number F33615-99-C-3611, by the Hungarian Fund for Scientific Research (OTKA) under contract $\mathrm{T}$ 017448, by the Hungarian Office of Higher Education Support Programs under contract FKFP 0654/2000, and by the Soros Foundation through a graduate research grant under contract $230 / 2 / 825$.

\section{References}

[1] Janos Sztipanovits, D. Michell Wilkes, Gabor Karsai, Csaba Biegl, and Lester E. Lynd, "The Multigraph and structural adaptivity," IEEE Transactions on Signal Processing, vol. 41, no. 8, pp. 2695-2716, August 1993.

[2] Gábor Péceli and Tamás Kovácsházy, "Transients in reconfigurable DSP systems," IEEE Trans. on Instrumentation and Measurement, vol. 48, pp. 986-989, October 1999.

[3] Vesa Välimäki and Timo I. Laakso, "Suppression of transients in variable recursive digital filters with a novel and efficient cancellation method," IEEE Transactions on Signal Processing, vol. 46, no. 12, pp. 3408-3414, December 1998.

[4] Alan B. Arehart and William A. Wolovich, "Bumpless switching in hybrid systems," in Hybrid Systems IV, Lecture Notes in Computer Science 1273, Antsaklis, Kohn, Nerode, and Shastry, Eds. 1997, Springer.

[5] Jeffrey. L. Musgrave, Ten-Huei Guo, Edmond Wong, and Ahmet Duyar, "Real-time accommodation of actuator faults on a reusable rocket engine," IEEE Trans. on Control System Technology, vol. 1, pp. 100-109, January 1997.

[6] Jovan D. Boskovic and Raman K. Mehra, "Stable multiple model adaptive flight control for accommodation of large class of control effector failures," in Proceedings of the 1999 American Control Conference, San Diego, California, USA, Jun 1999, vol. 3, pp. 1920-1924.

[7] Youmin Zhang and Jin Jiang, "Design of integrated fault detection diagnosis and reconfigurable control system," in Proceedings of the $3 \delta^{t h}$ IEEE Conference on Decision and Control, Phoenix, Arizona, USA, Dec 1999, vol. 4, pp. 3587-3592.

[8] Tamás Kovácsházy, Gábor Péceli, and Gyula Simon, "Transients in reconfigurable signal processing channels," in Proceedings of the 2000 IEEE Instrumentation and Measurement Technology Conference IMTC/2000, Baltimore, Md, USA, May 2000, vol. 1, pp. 241-245.

[9] Richard A. Roberts and Clifford T. Mullis, Digital Signal Processing, Addison Wesley, 1987.

[10] Mukund Padmanabhan, Ken Martin, and Gábor Péceli, Feedback-Based Orthogonal Digital Filters, Kluwer Academic Publishers, 1996.

[11] P. J. Mosterman and G. Biswas, "Diagnosis of continuous valued systems in transient operating regions," IEEE Trans. on Systems, Man and Cybernetics, vol. 29, pp. 554-565, Jun 1999. 